\title{
Computational inference of cancer-specific vulnerabilities in clinical samples
}

\author{
Kiwon Jang ${ }^{1 \dagger}$, Min Ji Park ${ }^{2 \dagger}$, Jae Soon Park', Haeun Hwangbo ${ }^{1}$, Min Kyung Sung ${ }^{1}$, Sinae Kim², Jaeyun Jung ${ }^{2}$, \\ Jong Won Lee ${ }^{3}$, Sei-Hyun $\mathrm{Ahn}^{3}$, Suhwan Chang ${ }^{2^{*}}$ and Jung Kyoon Choi ${ }^{1,4^{*}}$
}

* Correspondence: suhwan.chang@ amc.seoul.kr; jungkyoon@kaist.ac.kr ${ }^{\dagger}$ Kiwon Jang and Min Ji Park contributed equally to this work. ${ }^{2}$ Department of Biomedical Sciences, University of Ulsan College of Medicine, Asan Medical Center, Seoul 05505, Republic of Korea

'Department of Bio and Brain Engineering, KAIST, Daejeon 34141, Republic of Korea

Full list of author information is available at the end of the article

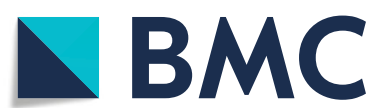

(c) The Author(s). 2020 Open Access This article is licensed under a Creative Commons Attribution 4.0 International License, which permits use, sharing, adaptation, distribution and reproduction in any medium or format, as long as you give appropriate credit to the original author(s) and the source, provide a link to the Creative Commons licence, and indicate if changes were made. The images or other third party material in this article are included in the article's Creative Commons licence, unless indicated otherwise in a credit line to the material. If material is not included in the article's Creative Commons licence and your intended use is not permitted by statutory regulation or exceeds the permitted use, you will need to obtain permission directly from the copyright holder. To view a copy of this licence, visit http://creativecommons.org/licenses/by/4.0/. The Creative Commons Public Domain Dedication waiver (http://creativecommons.org/publicdomain/zero/1.0/) applies to the data made available in this article, unless otherwise stated in a credit line to the data.

\section{Introduction}

There have been substantial efforts to profile cancer dependency by loss-of-function screens in cell lines [1-10], providing valuable resources that can lay foundation for new ways to fight cancer. However, the screening methods are applicable only to in vitro cell culture, limiting the discovery of therapeutic targets for clinical samples. A patient-derived cell model cannot be generated in a sizeable fraction of patients. In other patients, the time needed to develop the model is too long for clinical decisionmaking. Furthermore, in the absence of matched normal samples, it is difficult to identify truly cancer-specific dependencies and characterize them in association with somatic alterations.

Gene suppression will affect cell survival through the perturbation of the gene regulatory network. Perturbed transcriptomes, not basal transcriptomes, are directly linked to the consequential phenotypes. However, perturbed transcriptomes associated with 
cell death cannot be acquired experimentally because the expression patterns of only surviving cells will be captured. In addition, experimental data may be too complicated to capture the core changes that actually contribute to growth phenotypes because of biological complexity involving feedbacks and secondary effects. Practically, it is close to impossible to generate transcriptome data for knockdown of individual genes in each sample. Therefore, we need to simulate transcriptomic perturbations and link the resulting expression patterns to the state of cell death or growth.

To simulate all downstream events following gene suppression, we need a gene regulatory network represented as a directed graph encompassing all genes. In our previous work [11], we designed a comprehensive Bayesian prior model based on data for transcription factor binding, chromatin accessibility, enhancer-promoter interactions, and genetic expression association mapping. We then derived causal relationships from 1400 breast cancer transcriptomes. Another option is to assign orientations to links in the coexpression network, such as ARACNe (Algorithm for the Reconstruction of Accurate Cellular Networks) [12], that represents direct regulatory interactions.

In this work, we sought to develop a computational method that predicts cancerspecific dependencies for clinical samples with breast cancer as a model. We used the results of genome-wide shRNA [3] and CRISPR-Cas9 $[9,10]$ screening of breast cancer cell lines, the breast cancer regulatory networks described above, and the transcriptomic and mutational profiles of TCGA breast cancer samples [13]. Systematic characterization of common dependencies and experimental validation using patientderived cells identified novel therapeutic targets in breast cancer. This translational approach lays the foundation for future applications to discovering personalized therapeutic targets from clinical molecular data on various tumor types.

\section{Results}

Development of in silico CRISPR/RNAi and prediction model

The workflow of our method is illustrated in Fig. 1. Based on the gene regulatory network, we devised a method to simulate the perturbation effects of CRISPR or RNAi as detailed in the "Materials and methods" section. We employed the Bayesian network in breast cancer and the same type of network in liver cancer for a negative control from our previous works $[11,14]$. In this work, we constructed the ARACNe network [12] for breast and liver cancer. We determined the orientation of the links to simulate perturbation effects.

We merged two independent CRISPR-Cas9 screens of 28 and 25 breast cancer cell lines $[9,10]$, each based on a dependency score named CERES and BAGEL $[9,15]$, respectively. We selected three cutoffs (CERES $=-0.6,-1.0$, or -1.5 and BAGEL $=0,2$, or 4) to result in the similar number of dependencies per cell line between the two measures. As a result, the selected cutoffs led to 120,485, 44,644, and 3644 cases, respectively. From shRNA dropout screens of 77 breast cancer cell lines [3], each gene was assigned a normalized GARP (Gene Activity Ranking Profile) score, or zGARP, in each cell line $[3,16]$. Here, we used three cutoffs $(z G A R P=-2,-3$, or -4 ) to define dependencies, resulting in $88,837,31,229$, and 12,412 cases, respectively. We randomly chose the same number of instances below (for BAGEL) or above (for CERES and zGARP) the given cutoffs as independency cases. For each dependency and 


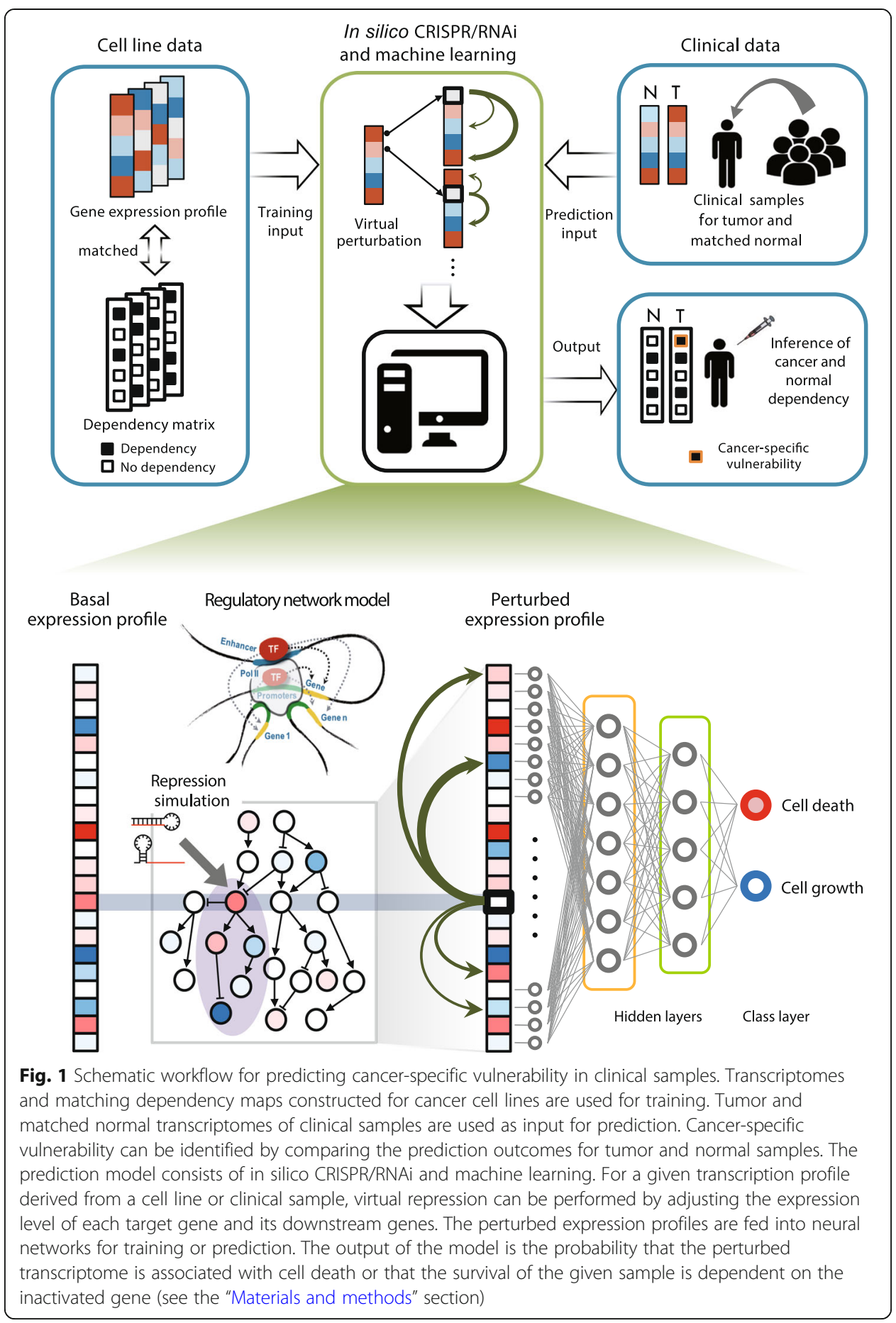

independency, we performed our in silico CRISPR/RNAi to generate the perturbed transcriptome.

We trained deep neural networks (DNN) with various hyperparameters (Additional file 2: Table S1) by using the perturbed expression patterns for the experimentally determined dependencies and independencies (Fig. 1). The ROC and precisionrecall curves show that better performance can be achieved with more stringent cutoffs for both CRISPR and RNAi with the Bayesian network (Fig. 2a and Additional file 1: 


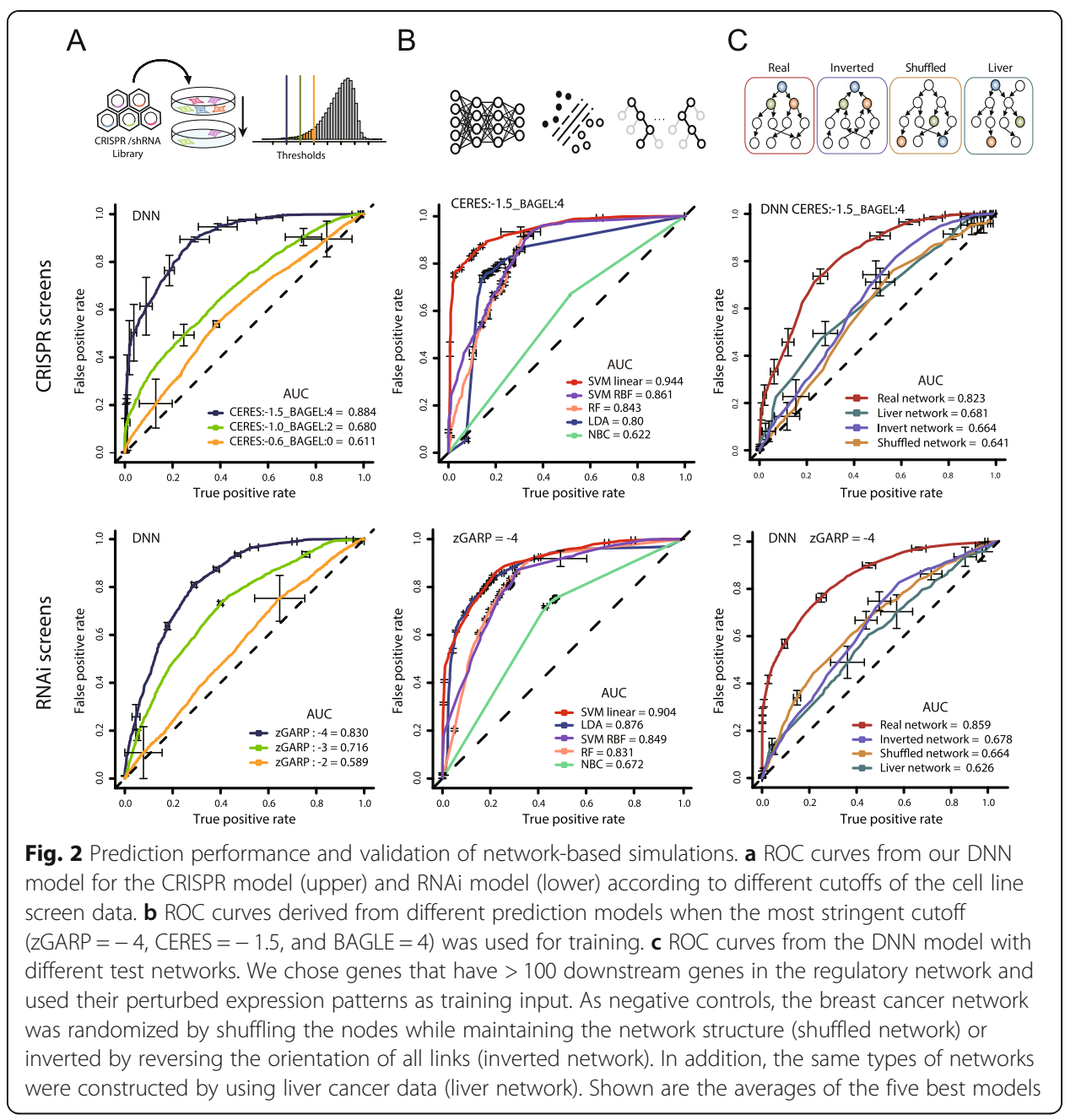

Figure S1A). These results imply that true dependencies share more distinct patterns in their perturbed transcriptomes. Similar performance results were obtained when we repeated the model training using the ARACNe network (Additional file 1: Figure S2A).

We also tested other machine learning methods including support vector machine (SVM) with different kernels, naïve Bayes classifier (NBC), linear discriminant analysis (LDA), and random forest (RF). When the most stringent cutoff was used, all methods except NBC showed acceptable performance in terms of AUC and AUPR with both loss-of-function screens and both regulatory networks (Fig. 2b, Additional file 1: Figures S1B, and S2). We tested the linear, polynomial, sigmoidal, and radial basis function (RBF) kernels for SVM. The polynomial and sigmoidal kernels presented performance levels similar to or lower than those of radial SVM.

Most importantly, we tested the validity of our network-based simulation. To this end, we chose genes that have $>100$ downstream genes in the network and used their perturbed expression patterns as training input. With these genes only, similar levels of prediction performance were achieved for both CRISPR and RNAi screens (red ROC curves in Fig. 2c). Three types of negative controls were prepared. First, we performed network randomization by shuffling the nodes while maintaining the network structure. Second, we generated inverted networks by reversing the orientations of all links. 
Third, we constructed networks in liver cancer to model biologically irrelevant regulatory interactions. Accurate predictions were not made in these cases (see green, violet, and yellow ROC curves in Fig. 2c, Additional file 1: Figures S1C, and S2C). In addition, the real network resulted in a significantly better agreement with experimental expression perturbation data in MCF7 (Additional file 1: Figure S3).

\section{Characterization of prediction outcomes for clinical samples}

We performed systematic in silico CRISPR/RNAi for 113 tumor and matched normal samples from TCGA and subjected the resulting expression profiles to our prediction models. First, we compared the TCGA prediction outcomes between the CRISPR and RNAi model. Strong positive correlations $(R=0.54 \sim 0.57)$ were observed between the screening models (Fig. 3a). Lower correlations $(R=0.24 \sim 0.26)$ were observed between

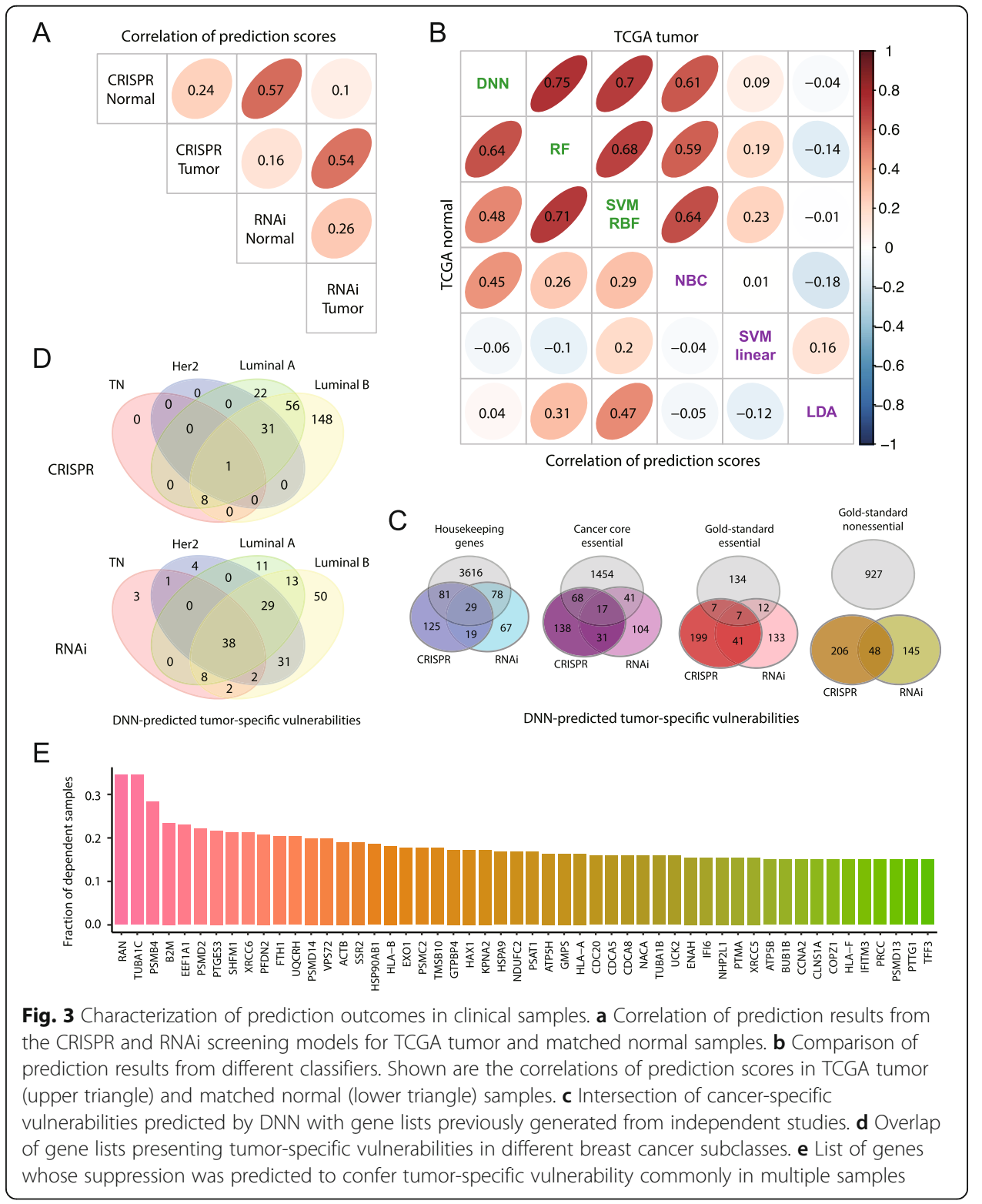


tumor and normal samples from the same donor (Fig. 3a). We also compared the different classifiers based on the RNAi model. In both tumor and normal samples, the two linear methods, LDA and linear SVM, did not agree with the other classifiers (Fig. 3b). The other linear classifier, NBC, showed positive correlations with the nonlinear classifiers (Fig. 3b). The three nonlinear methods, namely, DNN, radial SVM, and RF, presented highly consistent prediction results (Fig. 3b).

We identified genes that were predicted by DNN as dependencies in tumors but not in normal counterparts. According to a functional enrichment analysis [17], overrepresented functions included proteasome, amino acid biosynthesis, cell cycle, mRNA stabilization, oxidative phosphorylation, and post-translational protein modification (Additional file 3: Table S2). We compared the predicted cancer-specific dependencies with previously defined lists of genes such as the housekeeping genes [18], core fitness genes [1], and gold-standard essential or nonessential genes [19] (Fig. 3c). There were subclass-specific vulnerabilities (Fig. 3d).

We identified genes that were recurrently predicted as cancer-specific dependencies based on the average of the two screening models (Fig. 3e and Additional file 4: Table S3). Up to $35 \%$ of the samples were vulnerable to the loss of RAN. RAN encodes the small GTPase Ran, the key regulator of nucleocytoplasmic transport of proteins and ribonucleoproteins. Tumor-specific vulnerability to RAN suppression has been demonstrated in a few cell lines [20]. XRCC6 and XRCC5 encode the Ku heterodimer (Ku70/ $\mathrm{Ku} 80$ ), a main component of the nonhomologous end joining (NHEJ) pathway that repairs DNA double-strand breaks (DSBs). Proteasome subunits (PSMB4, PSMD2, PSMC2, PSMD14, PSMD13, and SHFM1) were also included in the list. PTGES3, a cochaperone functioning with heat shock protein 90 (HSP90), was identified together with other heat shock proteins such as HSP90AB1 and HSPA9. UQCRH, ATP5H, and NDUFC2 are involved in oxidative phosphorylation through respiratory electron transport. We conducted a detailed characterization of dependencies on Ran, Ku70/80, and proteasome subunits in the last section.

We then examined how our predictions for tumor samples differed from those for normal samples. The overall distribution of the dependency scores from different classifiers was compared between tumor and matched normal. In general, tumor samples presented high variability with biases toward high levels of dependencies (Fig. 4a). As discussed in the next section, these results imply that tumor undergoes extensive somatic changes that render the cells vulnerable to the loss of certain molecular activities. Among the nonlinear methods, DNN and radial SVM exhibited this pattern most clearly (Fig. 4a). RF showed this pattern only with the CRISPR model (Fig. 4a).

\section{Point mutations increase tumor-specific vulnerabilities}

Some cancer-specific vulnerabilities should be attributed to somatic DNA lesions that are only present in tumor cells. These can be either loss-of-function (LOF) or gain-offunction (GOF) mutations. When one of the two copies of a gene with an essential function is disrupted by LOF mutations, further suppression of this gene will lead to cell death in only cancer. However, this concept has been examined only for copy number loss in cell lines because matched normal controls are not available [21]. On the 


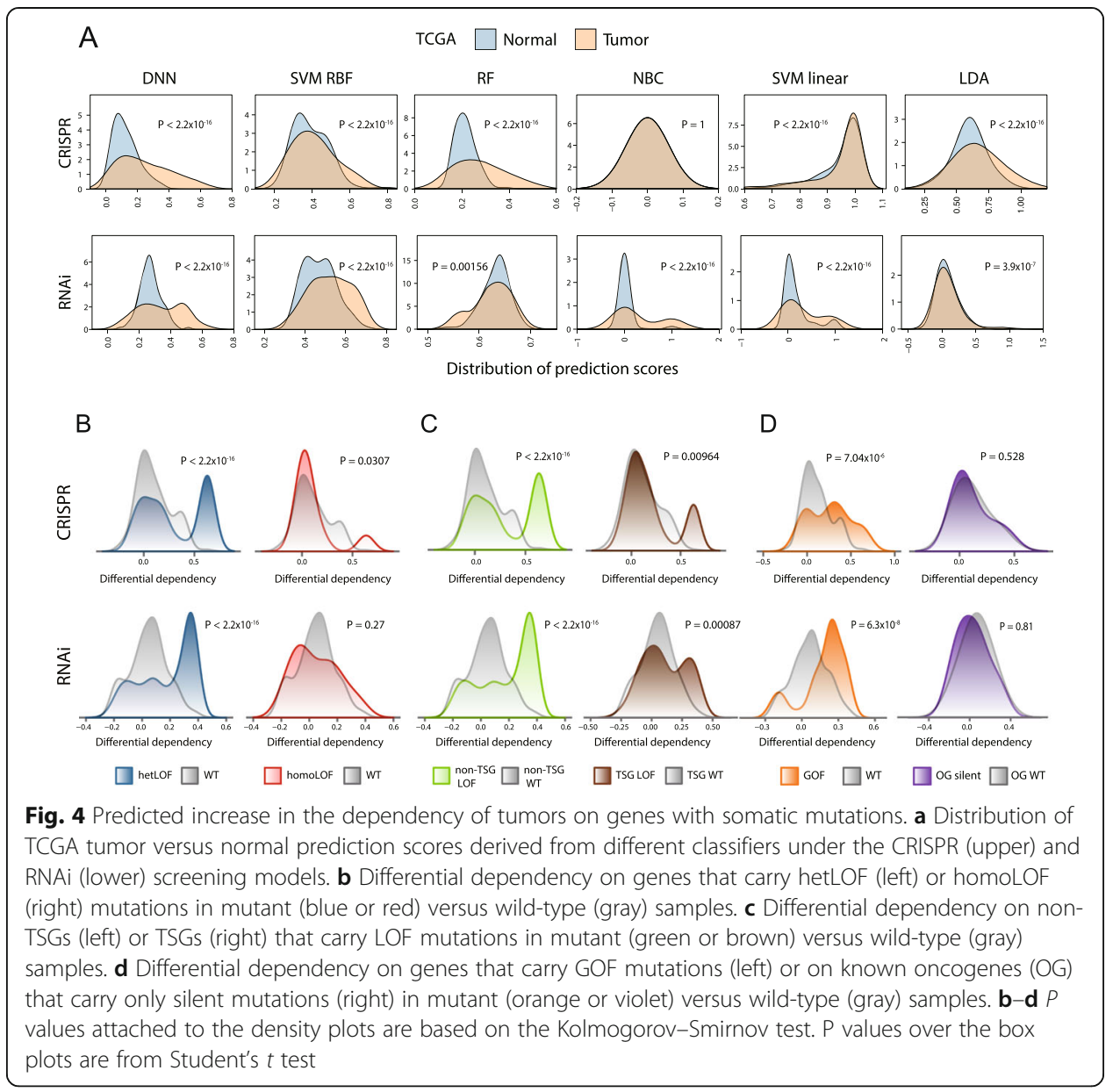

other hand, GOF mutations can be used to test oncogene dependence, a phenomenon also referred to as oncogene addiction [22].

We identified heterozygous LOF (hetLOF) and homozygous LOF (homoLOF) mutations. HomoLOF mutations may occur when an inactivation mutation arises in the remaining copy after hemizygous deletion. Also, we detected putative GOF mutations by looking for the cases in which the identical missense substitution was recurrently found across $\sim 1000$ breast cancer samples. We then segregated the samples according to mutation status. Then, the differential dependency on the mutated gene was obtained by subtracting the normal dependency score from the tumor dependency score.

We wanted to test whether our DNN was able to predict a relative increase in dependency on the mutated genes in tumors carrying the mutations compared to wildtype tumors. First, the differential dependency shifted to positive values specifically in samples with the hetLOF mutations (Fig. 4b, left). This pattern was not observed for samples in which both copies were already inactivated (Fig. 4b, right). Many tumor suppressor genes (TSGs) follow the two-hit hypothesis and contribute to tumorigenesis when both copies are lost or inactivated. Therefore, further suppression of these genes in LOF mutant samples will not cause tumor death. As expected, our DNN method predicted no acquired vulnerabilities for TSGs (Fig. 4c). Acquired dependencies on genes with GOF mutations, but not on oncogenes with silent mutations, were observed 
(Fig. 4d). With copy number alterations, there was no distinction between hemizygous versus homozygous deletion, non-TSGs versus TSGs, and oncogenes versus nononcogenes (Additional file 1: Figure S4). Therefore, point mutations may be a better indicator of acquired vulnerabilities than copy number alterations.

We tested the other classifiers in this respect. The nonlinear classifiers that made similar predictions as DNN, namely, radial SVM and RF, also showed acquired dependencies associated with the LOF or GOF mutations (Additional file 1: Figure S5). The only linear classifier producing results consistent with the nonlinear classifiers, NBC (Fig. 3b), was able to reproduce this pattern as well (Additional file 1: Figure S5). However, DNN showed most clear discrepancies between the mutants and wild-type samples.

\section{Predictions of context-dependent vulnerability or synthetic lethality}

In the previous section, we focused on vulnerabilities pertaining to mutations in the relevant gene itself. Some genes reveal context-dependent vulnerability under certain environmental stress or in particular genetic backgrounds such as cancer subtypes [1, 3]. Synthetic lethality arises when the simultaneous perturbation of two or more genes results in cell death [23]. Here, we examined whether our DNN model could predict context-dependent or synthetic vulnerabilities by focusing on two representative genes identified as common dependencies (Fig. 3e).

We first characterized the tumors that were predicted to be dependent on RAN based on the average of the CRISPR and RNAi model results. First, RAN itself and some RAN-related genes such as RANGAP1, RANBP1, RCC1, and TPX2 were overexpressed in these samples (Fig. 5a). Second, Her2-positive tumors, which grow faster than other subtypes, were significantly associated with RAN dependency (Fig. 5a). Third, functional enrichment of the overexpressed genes in the RAN-dependent samples was found for nuclear chromatin, ribosome, and spliceosome (Fig. 5a and Additional file 5: Table S4). In rapidly dividing tumors, proteins involved in chromatin formation need to be actively synthesized and transported into the nucleus. The assembly of ribosomes and spliceosomes is reliant on nucleocytoplasmic transport as the proteins are imported into the nucleus and then exported back to the cytosol after coupling with the RNAs. Ran is activated by growth signaling to fulfill this demand [24]. Therefore, rapidly growing tumors should be vulnerable to RAN silencing.

XRCC6 and XRCC5 encode Ku70 and Ku80 proteins, respectively. The Ku heterodimer recruits DNA-PKcs (encoded by PRKDC) to DSBs for DNA repair by the classical NHEJ pathway [25]. Upregulation of XRCC6 and other core NHEJ factors (e.g., PRKDC, LIG4, and XRCC4) was observed in the Ku-dependent tumors (Fig. 5b). Particularly significant upregulation was observed for ASF1A (Fig. 5b). This gene interacts with MDC1 to promote NHEJ repair [26]. MDC1 was also overexpressed (Fig. 5b). Two other mechanisms to repair DSBs are homologous recombination (HR) and alternative NHEJ. There is a synthetic lethal relationship between HR and classical NHEJ. HR-defective cells are sensitive to DNA-PKcs inhibition [27, 28]. MSH3 was identified as a novel HR pathway gene that significantly increases the sensitivity [28]. We observed a significant copy number loss of MSH3 and major HR genes (BRCA1, RAD50, and RAD51) in the Ku-dependent samples (Fig. 5b). Activation of alternative NHEJ was 


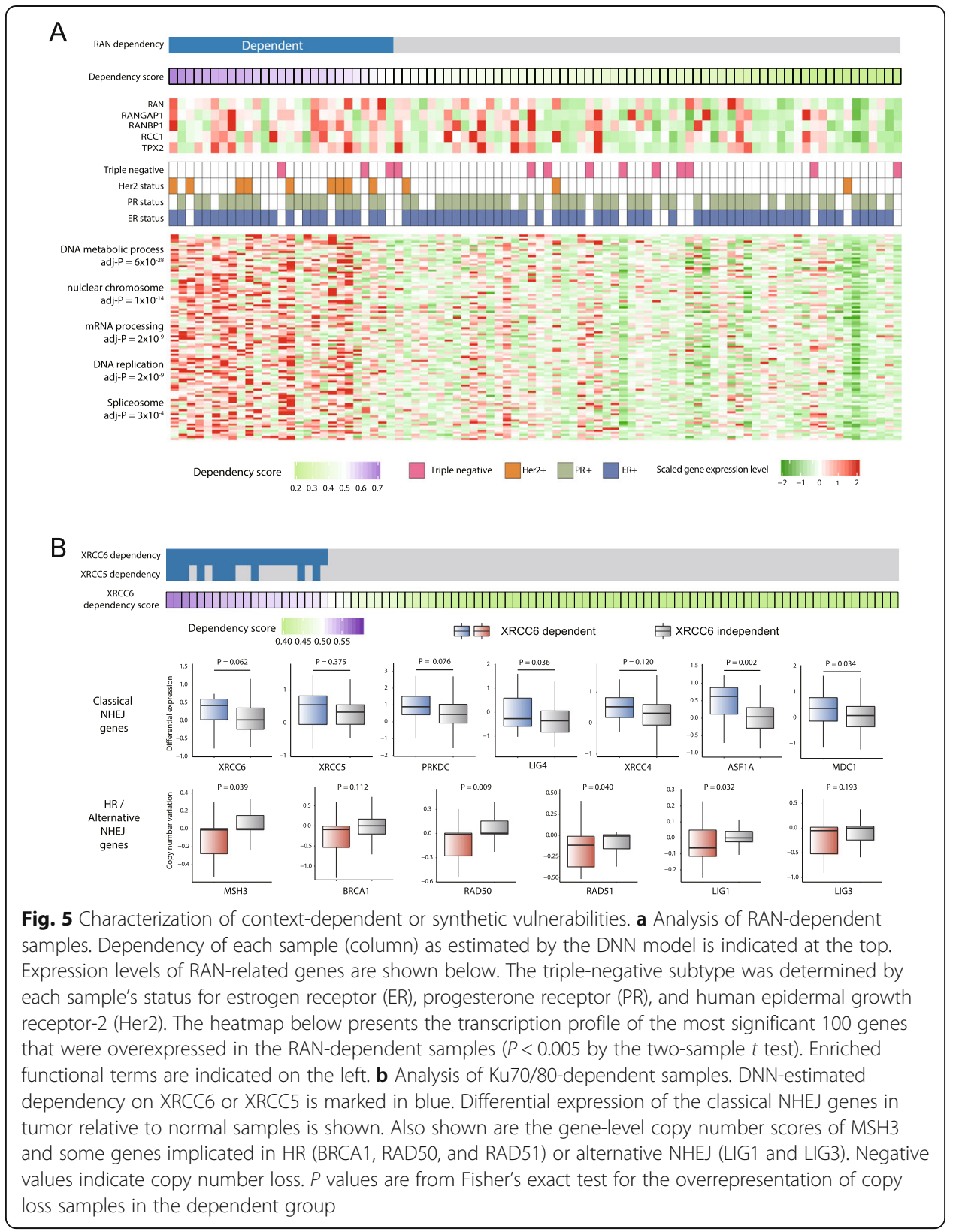

not observed in these tumors; whereas PARP1, XRCC1, MRE11, and RBBP8 (encoding CtIP) did not show upregulation (Additional file 1: Figure S6), some genes (LIG1 and LIG3) exhibited copy number loss (Fig. 5b).

\section{Experimental validation of predicted dependencies}

To test the applicability of our method in the clinical setting, we performed DNN prediction on the transcriptomes of our 24 patient-derived xenografts of breast cancer [29]. For RAN, 23 of the 24 tumors were predicted to be dependent. For experimental validation, we selected two samples whose predicted dependencies were positive on RAN and named them PD(+)RAN-1 and PD(+)RAN-2. For each of XRCC6 and PSMB4, we chose two 
samples with positive predicted dependencies and two with negative predicted dependencies: $\mathrm{PD}(+/-) \mathrm{XRCC6/PSMB4-1/2}$. We derived patient-derived cells for these samples; performed CRISPR-Cas9 [30] targeting of RAN, XRCC6, or PSMB4; and confirmed the stable expression of Cas9 and the reduced expression of the target genes.

RAN suppression in the $\mathrm{PD}(+) \mathrm{RAN}$ cells resulted in an increase in the proportion of Annexin V-positive cells, indicating the induction of apoptosis, and a decrease in the fraction of proliferating cells according to the BrdU assay (Fig. 6a and Additional file 1: Figure S7). For XRCC6 and PSMB4 silencing, the induction of apoptosis and the decrease of cell proliferation were remarkably more pronounced in the $\mathrm{PD}(+)$ cells than in the PD(-) cells (Fig. 6b, c and Additional file 1: Figures S8 S9). These results demonstrate that our method to predict sample-specific vulnerabilities can lead to the discovery of precision therapeutic targets.

Next, we explored the mechanisms underlying cell death induced by the inactivation of these genes. In the $\mathrm{PD}(+) \mathrm{RAN}$ cells, we tested the nuclear translocation of epidermal growth factor receptor (EGFR). The ligand EGF stimulates the translocation of phosphorylated EGFR (pEGFR) to the nucleus [31]. The EGFR nuclear transport was reported to be RANdependent [32]. When RAN was inactivated by CRISPR/Cas9, the nuclear fraction of pEGFR vanished whereas cytoplasmic pEGFR was not affected (Fig. 7a, b and Additional file 1: Figure S10). This resulted in the downregulation of two important transcriptional targets of pEGFR, that is, Cox2 and Cyclin D1 (Fig. 7a). For PSMB4, we tested the degradation of proapoptotic proteins. PSMB4 knockdown increased the protein levels of Bad, Bim, and Cytochrom C in the PD(+)PSMB4 cells (Additional file 1: Figure S11).

Regarding Ku70/80 dependency, we first tested whether the PD(+)XRCC6 cells rely on the NHEJ pathway. When XRCC6 was inactivated, the $\mathrm{PD}(+)$ cells maintained the activity of DNA-PKcs whereas the $\mathrm{PD}(-)$ cells decreased the expression of this protein (Additional file 1: Figure S12). Indeed, the $\mathrm{PD}(+)$ samples were more sensitive to the DNA-PK inhibitor, NU-7026, as indicated by $40-60 \%$ lower IC50 values (Additional file 1: Figure S13A S13B). This reliance on the NHEJ pathway may reflect HR deficiency. It is known that HR-defective cells are sensitive to DNA-PKcs inhibition [27, 28]. To test this, we challenged the $\mathrm{PD}(+/-)$ XRCC6 cells with $\gamma$-irradiation to generate DSBs. XRCC6 inactivation led to an increase in the number of Rad51 foci in both the PD(+) and $\mathrm{PD}(-)$ cells (Fig. 7c). However, the degree of the increase of Rad51 foci was 3 4fold lower in the $\mathrm{PD}(+)$ cells than in the $\mathrm{PD}(-)$ cells (Fig. $7 \mathrm{~d}$ ). We also performed the DR-GFP/I-SceI HR assay [33] in which the fraction of GFP-positive cells indicates HR activity for DSB repair. While XRCC6 suppression led to an increase in the fraction of the GFP-positive cells (Fig. 7e), the magnitude of increase was 3 6-fold lower in the $\mathrm{PD}(+)$ cells than in the $\mathrm{PD}(-)$ cells (Fig. 7f). The similar results were obtained from the DR-GFP/I-SceI assay in response to the treatment of NU-7026 (Additional file 1: Figure $\mathrm{S} 13 \mathrm{C})$. In summary, the PD(+)XRCC6 cells displayed HR deficiencies under high DSB load, consistent with our observation with the TCGA data (Fig. 5b). These results illustrate how Ku70/80 silencing can render particular tumors vulnerable when the sensitivity to Ku70/80 suppression can be predicted by our computational method.

\section{Discussion}

There was an attempt to develop a predictive model on the basis of cell line screening data [4]. This model was reliant on common molecular markers of cell lines with 
A
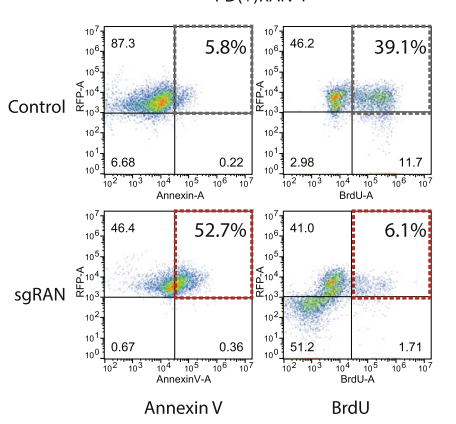

B
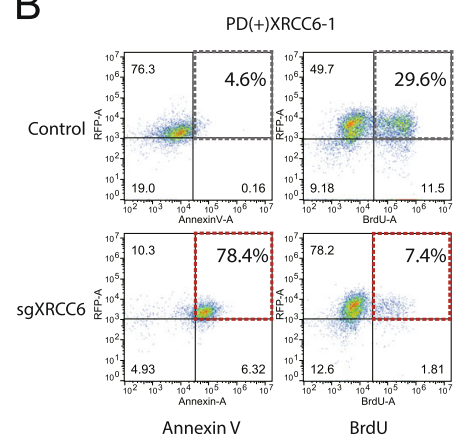

C
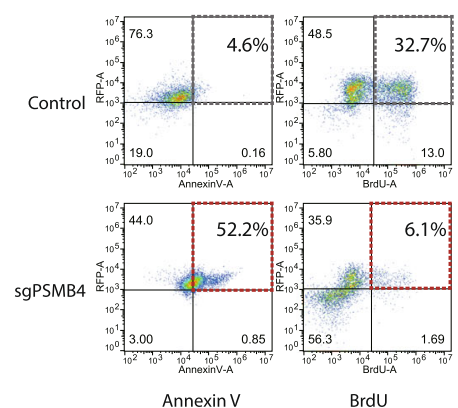
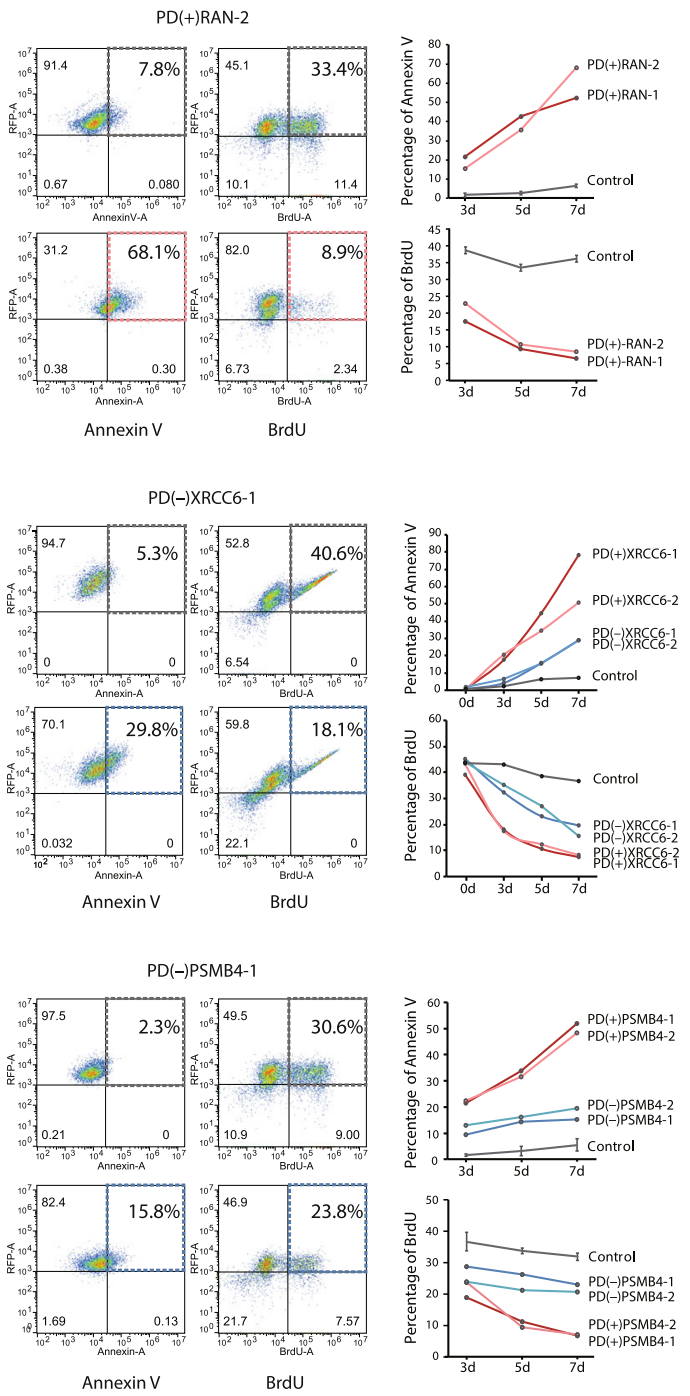

Fig. 6 Experimental validation of predicted vulnerabilities by apoptosis and cell proliferation measurements. a Scatter plots showing the distribution of Annexin $V$ and BrdU staining, which marks apoptosis and cell proliferation, respectively, for two patient-derived cells with positive predicted dependency on RAN, namely, PD(+)RAN-1 and PD(+)RAN-2, before and after RAN inactivation by CRISPR-Cas9. Shown here are day 7 results. Plots for days 3 and 5 are provided in Additional file 1: Figure S7. The graphs on the right indicate the percentage of Annexin V- or BrdU-positive cells according to days. b, c Scatter plots showing the distribution of Annexin $V$ and BrdU staining for patient-derived cells with positive or negative predicted dependency on $\mathbf{b}$ XRCC6 and c PSMB4 before and after CRISPR-Cas9 knockout. Shown here are day 7 results for PD(+)XRCC6-1, PD(-)XRCC6-1, PD(+)PSMB4-1, and PD(-)PSMB4-1. Plots for days 3 and 5 after inactivation in these cells, and data for days 3, 5, and 7 after inactivation in PD(+)XRCC6-2, PD(-)XRCC6-2, PD(+)PSMB4-2, and PD(-)PSMB4-2 are provided in Additional file 1: Figures S8 S9. The graphs on the right indicate the percentage of Annexin $\mathrm{V}$ - or BrdU-positive cells according to days after inactivation

shared dependencies. In this case, the number of available cell lines for each tumor type, and thus the number of dependent cases to be learned, is limited. Moreover, this approach is applicable only to context-dependent variability or synthetic lethality, and predictions cannot be made for sample-specific dependencies.

Instead of using basal expression patterns as predictors, we performed simulations to obtain transcriptome patterns resulting from gene suppression. Because these 


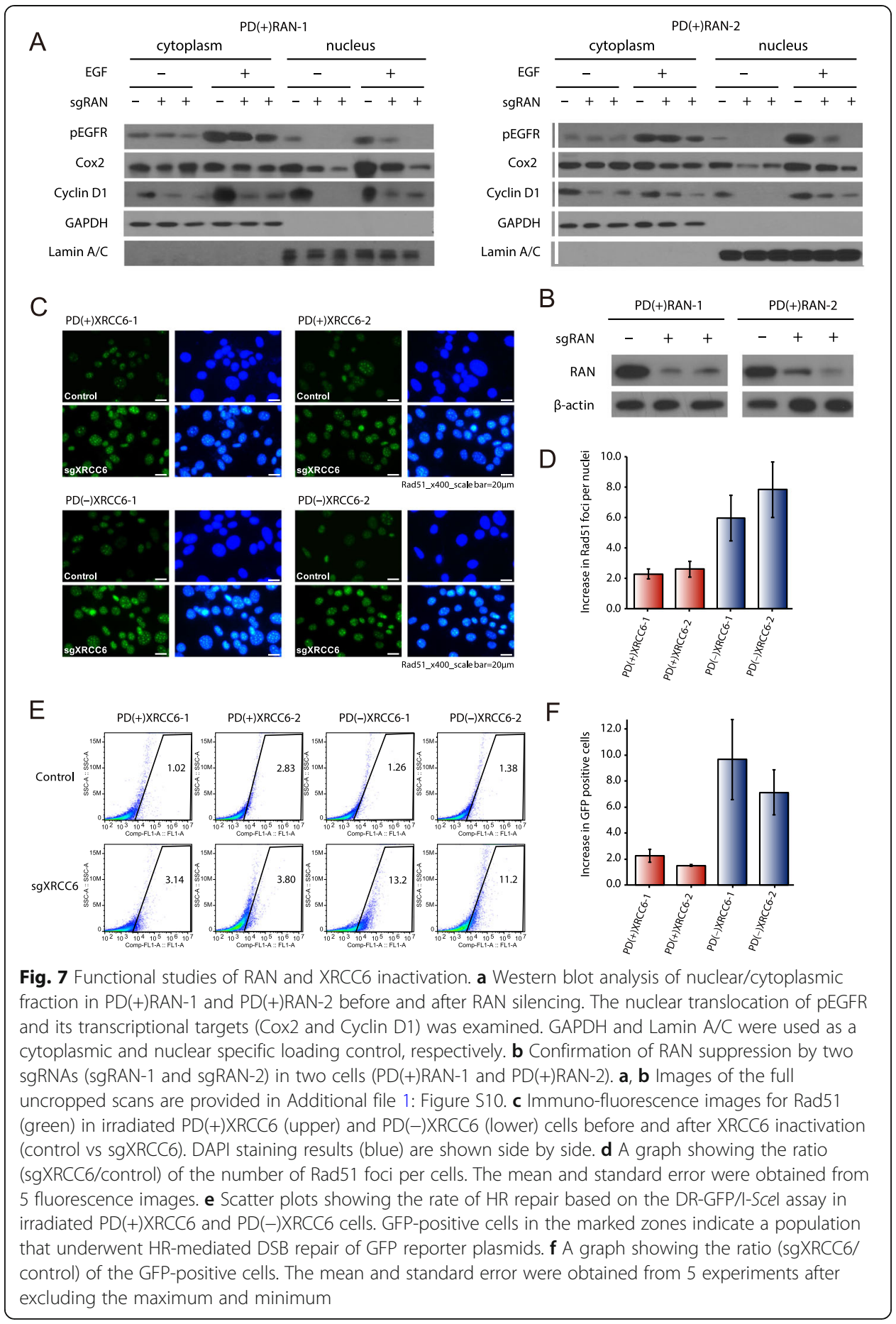

simulations could be repeated for individual genes, as many perturbed profiles as genes could be obtained from one basal profile, which cannot be accomplished experimentally. Therefore, a sufficient number of cases could be used to train predictive models. Also, it is difficult to obtain experimental profiles associated with cell death because the expression patterns of only living cells can be captured.

The three genes we tested experimentally are presented as common essential genes in the depmap portal browser (https://depmap.org/portal). In contrast, our model 
predicts that they are dependencies in 20 30\% of tumors (Fig. 3e). As for XRCC6 and PSMB4, this seems to result from the different scoring scheme used in the browser. The scoring metric used in our model indicates similar fractions of dependent cell lines at the threshold we used, thereby emphasizing consistencies between our prediction and cell line screening results. As for RAN, we characterized the gene as a contextdependent dependency in association with cell growth (Fig. 5a). This may explain why RAN is detected as a common dependency in cell line screening in which rapidly growing cells dominate the culture population. This highlights the advantage of our model over screening results.

A classic example of synthetic lethality is the interaction between poly (ADP-ribose) polymerase 1 (PARP1) and BRCA1/2 [34-36]. Later, the sensitivity of BRCA1/2-mutated tumors to PARP inhibitors was broadly linked to HR repair defects [23]. Further studies established synthetic lethality between the HR pathway and the NHEJ gene DNA-PKcs [27, 28, 37, 38]. Our results suggest that HR-defective tumors are also sensitive to the loss of Ku70/80, the interacting partner of DNA-PKcs. Further investigation is required as to whether inhibiting Ku70/80 instead of DNA-PKcs can be more effective at the RNA level. Importantly, sensitivity to Ku70/80 inhibition can be predicted based on transcriptome data by our deep learning model.

This approach can be extended to many different cancer types, facilitating the discovery of precision therapeutic targets. Once a prediction model has been established, exome and transcriptome data for tumor and matched normal samples will be sufficient to identify patient-specific vulnerabilities that are associated with somatic mutations or common vulnerabilities that arise in a context-dependent or synthetic lethal manner.

\section{Materials and methods}

\section{Cell line dependency screening data}

We downloaded two independent CRISPR-Cas9 dropout screening results for 28 and 25 breast cancer cell lines $[9,10]$ from https://depmap.org/portal/download and https://score.depmap.sanger.ac.uk/downloads. Genetic dependencies were measured based on the CERES [9] and BAGEL [15] scores, respectively. We selected different cutoff values (CERES $=-0.6,-1.0$, or -1.5 and BAGEL $=0,2$, or 4 ) to result in the similar number of dependencies per cell line between the two datasets. In addition, we obtained shRNA dropout screening results for 77 breast cancer cell lines [3] from http://neellab.github.io/bfg. Normalized GARP score, or zGARP, was used as a metric of dependency $[3,16]$. Here, a zGARP of $-2,-3$, or -4 was used as the threshold to define experimentally determined dependency. Gene expression profiles of the cell lines were also obtained from the same websites.

\section{Regulatory network construction}

For regulatory network construction, we downloaded TCGA RNA-seq data for 1215 breast cancer and 423 liver cancer samples from the UCSC Cancer browser (https:// xenabrowser.net). We employed our previous Bayesian network in breast cancer [11] (Additional file 6: Table S5) and the same type of network in liver cancer [14] for a negative control. We constructed another type of regulatory network on the basis of ARACNe (Algorithm for the Reconstruction of Accurate Cellular Networks) [12] 
(Additional file 7: Table S6). We applied the ARACNe software available at http://califano.c2b2.columbia.edu/aracne [39]. From a gene expression profile, ARACNe identifies direct transcriptional interactions on the basis of mutual information (MI). We determined the significance of MI at $P=0.01$. In order to simulate the effect of gene suppression, we had to determine the directionality (i.e., which gene regulates which gene) and regulation type (i.e., whether the interaction is activation or inhibition) of the interactions between the suppression target gene and its interaction partners. For this purpose, we utilized conditional probability. For example, when $\mathrm{P}(\mathrm{X}=\mathrm{on} \mid \mathrm{Y}=\mathrm{on})$ represents the probability that gene $\mathrm{X}$ is expressed given that gene $\mathrm{Y}$ is expressed, the directionality can be determined by comparing $\mathrm{P}(\mathrm{X}=$ on $\mid \mathrm{Y}=$ on $)$ and $\mathrm{P}(\mathrm{Y}=$ on $\mid \mathrm{X}=$ on $)$. If $\mathrm{P}(\mathrm{X}=$ on $\mid \mathrm{Y}=\mathrm{on})>\mathrm{P}(\mathrm{Y}=$ on $\mid \mathrm{X}=$ on $)$, gene $\mathrm{Y}$ is considered to be a regulator of gene $\mathrm{X}$. To determine the regulation type, we used $\mathrm{P}(\mathrm{X}=\mathrm{up} /$ down $\mid \mathrm{Y}=\mathrm{up} /$ down $)$ to represent the probability of gene $\mathrm{X}$ being up- or downregulated given the up- or downregulation of gene Y. More details are provided in the next section.

\section{In silico CRISPR/RNAi methodologies}

The first step of our in silico CRISPR/RNAi was to identify genes whose expression level would be affected by the suppression of the target gene. From the Bayesian network, all descendant genes were identified by traveling via outgoing links from the target gene. We determined only whether a given gene is a descendant of the target gene while ignoring the structure of the intervening nodes between the two genes. Then, the expression level of each descendant was adjusted depending on the sign and strength of its expression correlation with the target gene. Here, we denote the target gene as gene $\mathrm{Y}$ and the $j$ th descendant of gene $\mathrm{Y}$ as gene $\mathrm{X}_{j}$. When $x_{j}$ and $y$ indicate the basal expression level of $\mathrm{X}_{j}$ and $\mathrm{Y}$ in the given sample, respectively, the perturbed expression level of the $j$ th gene, $x_{j}^{\prime}$, can be obtained as

$$
x_{j}^{\prime}=x_{j}-r_{j} \frac{y-y^{\prime}}{y} x_{j},
$$

where $r_{j}$ is the correlation coefficient of the expression levels of gene $\mathrm{X}_{j}$ and $\mathrm{Y}$ across reference samples, and $y^{\prime}$ is the perturbed expression level of the target gene, Y. For reference samples, we used the same transcriptome data that were used for network construction. We set $y^{\prime}=0$ for CRISPR simulation and $y^{\prime}=0.2 y$ for RNAi considering the known average experimental efficacy of shRNA [40]. Instead of repeating this calculation gene by gene, we utilized matrix multiplication. The perturbed expression level of the $j$ th gene by the $i$ th target gene was obtained as the $(i, j)$ th entry of the perturbed expression matrix $P$ as follows.

$$
\begin{aligned}
& P_{i, j}=-0.8(R \cdot B)_{i, j}+B_{j, j} \\
& \text { where } R=\left[\begin{array}{ccc}
1 & \cdots & r_{n} \\
\vdots & \ddots & \vdots \\
0 & \cdots & 1
\end{array}\right] \text { and } B=\left[\begin{array}{ccc}
x_{1} & \cdots & 0 \\
\vdots & \ddots & \vdots \\
0 & \cdots & x_{n}
\end{array}\right]
\end{aligned}
$$

$R$ is an adjacency matrix for expression correlations and $B$ is a basal expression matrix filled with zero except at the diagonal. 
For the ARACNe network, we utilized the conditional probability instead of the correlation coefficient. First, all the first neighbors of the target gene were identified. We denote the target gene as gene $\mathrm{Y}$ and the $j$ th neighbor of gene $\mathrm{Y}$ as gene $\mathrm{X}_{j}$. Among them, the regulatory targets, not the regulators, of gene $\mathrm{Y}$ were determined based on the following set of conditional probabilities.

$$
\begin{gathered}
\mathrm{P}\left(\mathrm{X}_{j}=\text { activator }\right)=\frac{P\left(\mathrm{Y}=\text { upn } \mathrm{X}_{j}=\mathrm{up}\right)+P\left(\mathrm{Y}=\text { down } n \mathrm{X}_{j}=\text { down }\right)}{P\left(\mathrm{X}_{j}=\mathrm{up}\right)+P\left(\mathrm{X}_{j}=\text { down }\right)} \\
\mathrm{P}(\mathrm{Y}=\text { activator })=\frac{P\left(\mathrm{X}_{j}=\mathrm{up} \cap \mathrm{Y}=\mathrm{up}\right)+P\left(\mathrm{X}_{j}=\text { down } \cap \mathrm{Y}=\text { down }\right)}{P(\mathrm{Y}=\mathrm{up})+P(\mathrm{Y}=\text { down })} \\
\mathrm{P}\left(\mathrm{X}_{j}=\text { inhibitor }\right)=\frac{P\left(\mathrm{Y}=\text { down } \mathrm{X}_{j}=\mathrm{up}\right)+P\left(\mathrm{Y}=\text { upn } \mathrm{X}_{j}=\text { down }\right)}{P\left(\mathrm{X}_{j}=\mathrm{up}\right)+P\left(\mathrm{X}_{j}=\text { down }\right)} \\
\mathrm{P}(\mathrm{Y}=\text { inhibitor })=\frac{P\left(\mathrm{X}_{j}=\text { down } \cap \mathrm{Y}=\mathrm{up}\right)+P\left(\mathrm{X}_{j}=\text { up } \cap \mathrm{Y}=\text { down }\right)}{P(\mathrm{Y}=\mathrm{up})+P(\mathrm{Y}=\text { down })}
\end{gathered}
$$

Up- and downregulation was defined using the reference transcriptome samples that were used for network construction. For each gene, the mean expression level, $\mu$, and its standard deviation, $\sigma$, were obtained across the reference samples. We set $\mathrm{X}_{j}=$ up and $\mathrm{Y}=$ up when their expression level in the given reference sample was larger than $\mu+\sigma$. On the contrary, we set $\mathrm{X}_{j}=$ down and $\mathrm{Y}=$ down when their expression level in the given reference sample was lower than $\mu-\sigma . \mathrm{X}_{j}$ was considered to be a regulatory target of $\mathrm{Y}$ when $\mathrm{P}\left(\mathrm{X}_{j}=\right.$ activator $)+\mathrm{P}\left(\mathrm{X}_{j}=\right.$ inhibitor $)<\mathrm{P}(\mathrm{Y}=$ activator $)+\mathrm{P}(\mathrm{Y}=$ inhibitor $)$. Once $\mathrm{X}_{j}$ has been determined as a target of $\mathrm{Y}$, the regulation type of the link from $\mathrm{Y}$ to $\mathrm{X}_{j}$ was determined by comparing $\mathrm{P}(\mathrm{Y}=$ activator $)$ and $\mathrm{P}(\mathrm{Y}=$ inhibitor $)$. Finally, when $x_{j}$ and $y$ indicate the basal expression level of $\mathrm{X}_{j}$ and $\mathrm{Y}$, respectively, in a given sample subjected to in silico CRISPR/RNAi, the perturbed expression level of the $j$ th gene, $x_{j}^{\prime}$, was obtained as

$$
x_{j}^{\prime}=\left\{\begin{array}{c}
x_{j}-\mathrm{P}(\mathrm{Y}=\text { activator }) \frac{y-y^{\prime}}{y} x_{j}, \text { if } \mathrm{P}(\mathrm{Y}=\text { activator })>\mathrm{P}(\mathrm{Y}=\text { inhibitor }) \\
x_{j}+\mathrm{P}(\mathrm{Y}=\text { inhibitor }) \frac{y-y^{\prime}}{y} x_{j}, \text { if } \mathrm{P}(\mathrm{Y}=\text { activator })<\mathrm{P}(\mathrm{Y}=\text { inhibitor })
\end{array}\right.
$$

For CRISPR/RNAi target genes, we considered all genes in the network, except those at the terminal nodes, when the expression data was available in the basal transcriptome that is subjected to in silico CRISPR/RNAi. We repeated the above procedures for all available genes. In a test for the influence of the in silico CRISPR/RNAi processes on prediction performance, we chose genes that have $>100$ descendants or regulatory targets in the network and used their perturbed expression patterns as training input. We performed network randomization by shuffling the nodes while maintaining the network structure. We also generated inverted networks by reversing the directions of all links to test the validity of inferred regulator-target relationships.

In breast cancer, the Bayesian regulatory network consisted of 13,047 genes, and the ARACNe network was composed of 8463 genes. Among them, 7439 and 7086 genes in the Bayesian network and ARACNe network, respectively, turned out to regulate at least one gene. Therefore, we were able to perform in silico CRISPR/RNAi for these genes only. 


\section{Deep neural network modeling}

We developed a deep neural network (DNN) model by using the Theano library [41]. DNN architecture expresses complex nonlinear relationships embedded in input features through multiple layers of artificial neural networks. However, a large number of manual attempts needs to be tested because model performance is sensitive to many calibration parameters or hyperparameters. Here, we used three types of calibration parameters: (i) parameters for model architecture, such as the number of hidden layers and number of hidden nodes; (ii) parameters for model algorithm, such as the learning rate, momentum, batch size, activation function, and initial weight distribution; and (iii) parameters to avoid overfitting, such as regularization parameters (L1 and L2) and dropout rate. The calibration parameters we used in this work are summarized in Additional file 2: Table S1.

Pre-training processes can lead to optimal initial points of weight and bias, thereby reducing the chance to fall into local minima [42]. We constructed a stacked denoising autoencoder (SdA) with the same structure of the DNN model except that the output layer has the same number of nodes as the input layer. Through a process known as denoising, we generated a stochastically corrupted version of the log-normalized input vector $\mathrm{x}$ consisting of the perturbed expression levels of $n$ genes, $\mathrm{x} \in[0,1]^{n}$. The SdA mapped the corrupted $\mathrm{x}$ to a hidden layer $\mathrm{y}, \mathrm{y} \in[0,1]^{m}$, using the activation function $f$. This encoding process can be expressed as

$$
\mathrm{y}=f(\mathrm{Wx}+\mathrm{b})
$$

where $\mathrm{W}$ is the weight matrix and $\mathrm{b}$ is the bias. The following decoding step generated the reconstruction vector $\mathrm{z}$ given as

$$
\mathrm{z}=f\left(\mathrm{~W}^{T} \mathrm{y}+\mathrm{b}^{\prime}\right)
$$

while minimizing reconstruction error represented as Cost. Cost was defined as follows according to the activation function of the DNN model:

1. If the ReLU function was used,

$$
\text { Cost }=\frac{1}{B} \sum_{k=1}^{B}\left(x_{k}-z_{k}\right)^{2} .
$$

2. If the sigmoid function was used,

$$
\text { Cost }=-\frac{1}{B} \sum_{k=1}^{B}\left[x_{k} \log z_{k}+\left(1-x_{k}\right) \log \left(1-z_{k}\right)\right] .
$$

Here, $B$ indicates a batch size. Some entries of the input vector $\mathrm{x}$ were masked according to the dropout rate. Parameter $\theta$ (weight and bias) was updated through the stochastic gradient descent at each training epoch step as

$$
\theta_{t+1}=\theta_{t}-\alpha \nabla_{\theta_{t}}
$$

where $t$ indicates training epochs.

To further prevent overfitting, we applied early stopping and feature selection.

1. Early stopping is a form of regularization used to avoid overfitting by stopping training at points where performance does not improve. One fifth of the training 
data were used for performance evaluation. Three fourths of the remaining were used for model learning, and the remaining 1/4 were used for stop control. All models were trained only to the point when performance reached a plateau. To determine the stopping point while avoiding overfitting, the training set was further divided into two independent parts, one for model training and the other for performance tracing. We defined a variable called patience and initially set it to 200. We then trained the model while keeping the number of training epochs below patience. Whenever performance improvement was observed, we updated patience to $\max ($ initial patience, current epoch $\times 2$ ) and saved the current model as the best. The threshold of improvement was set to 0.99 .

2. To prevent overfitting due to an excess of learning features, we reduced the number of genes whose perturbed expression levels were used as input. Specifically, we selected 1000 genes whose expression values varied most across different cases (perturbed transcriptomes) based on standard deviation.

After pre-training, we fine-tuned the model to minimize the loss function defined as

$$
\text { Loss }=\mathrm{NLL}+\lambda_{1}\|w\|_{1}+\lambda_{2}\|w\|_{2}
$$

where NLL denotes the mean of negative log likelihood, and $\lambda_{1}|| w\left\|_{1}+\lambda_{2}|| w\right\|_{2}$ represents the regularization term of the elastic net that is used to control overfitting. $\|\cdot\|_{p}$ indicates the $L_{p}$ norm and was given as

$$
\|w\|_{p}=\left(\sum_{j=0}^{|w|}\left|w_{j}\right|^{p}\right)^{\frac{1}{p}}
$$

where $\lambda_{p}$ is a hyperparameter that controls the relative contribution of each regularization term. As implied in the above formula, the elastic net is a combination of lasso $\left(L_{1}\right)$ and ridge $\left(L_{2}\right)$ regularization on the weights of the model. The elastic net is known to outperform $L_{1}$ or $L_{2}$ regularization alone and to be especially useful when the number of predictors is large [43]. The $N L L(\theta)$ of the loss function was given as

$$
N L L(\theta)=-\frac{1}{B} \sum_{i=1}^{B}\left(Y^{i} \log f(\theta)^{i}+\left(1-Y^{i}\right) \log \left(1-f(\theta)^{i}\right)\right),
$$

where $f(\theta)^{i}$ is an output for the $i$ th in silico CRISPR/RNAi target gene in a mini-batch of size $B$. Each target, $Y$, can be either 1 or 0 where 1 indicates that there is dependency on the gene in the given sample. The loss function was composed of parameters $(\theta)$ including $w_{m}^{k}, b_{k}, w_{k}$, and $b^{\prime}$, which were updated by the standard backpropagation algorithm with momentum. The momentum method is a technique to accelerate the gradient descent process by accumulating a velocity $(v)$ in the direction of stable and persistent reduction of the loss function [44]. For the given loss function, the momentum was given as

$$
\begin{aligned}
& \theta_{t+1}=\theta_{t}+v_{t+1}, \\
& v_{t+1}=\mu v_{t}-\varepsilon \nabla\left(\operatorname{Loss}\left(\theta^{t}\right)\right),
\end{aligned}
$$

where $\varepsilon$ is the learning rate, $\mu$ is the momentum coefficient, and $\nabla\left(\operatorname{Loss}\left(\theta^{t}\right)\right)$ is the gradient at $\theta^{t}$. $v_{0}$ was set to 0 . 


\section{Model construction}

While evaluating prediction performance on a total of 93,312 combinations of hyperparameter values (Additional file 2: Table S1), we observed that performance does not improve considerably after 1000 trials in general. Therefore, we used 1000 random combinations of the parameter values for model selection. The learning processes were based on five-fold cross-validation consisting of five different partitions into training and validation data. For each partition of training and validation data, the best hyperparameter combination was selected based on the AUC on the validation data set. A test AUC was then assigned to the selected model based on an independent test set that was not used for learning. The average test AUC for the five best models was obtained to represent the overall performance. In our tests of the shuffled, inverted, and liver cancer networks, we selected the five best hyperparameter combinations for each split into a training set and a validation set, resulting in five average test AUC values. The mean of these five values was reported. We used the class probability as the "prediction score" for the given input. A prediction score close to 1 indicates a higher association of the given transcriptome with cell death. The input transcriptome with a prediction score greater than 0.5 was classified as a dependency profile. Tumor-specific dependencies were identified as having the tumor prediction score greater than 0.5 and the normal prediction score lower than 0.5 .

\section{Other classifiers}

We also implemented random forest [45], support vector machine [46], naïve Bayes classifier [46], and linear discriminant analysis [47]. We tested the linear, polynomial, sigmoid, and radial basis kernels for the support vector machine. By using the Cclassification type, we used the support vector machine as a classification machine. Random forest was trained with 1000 decision trees by using the $\mathrm{R}$ package "randomForest." We used the R package "e1071" to implement support vector machine and naïve Bayes classifier. The R package "MASS" was used to implement linear discriminant analysis. A prior probability of 0.5 was given to the two classes (dependency versus independency).

\section{Analysis of TCGA breast cancer data}

We downloaded the expression profiles of 1095 tumor samples along with those of 113 matched normal samples from the TCGA database [13]. Somatic variant calls from whole-exome sequencing for 988 cases were also obtained. Of these, the matched normal expression profiles were available for 110 samples. For copy number analysis, we obtained the gene-level GISTIC2.0 scores [48], which were available for 111 samples. Copy number variations for genomic segments were mapped to protein-coding genes by the TCGA FIREHOSE pipeline. The gene-level GISTIC2.0 scores were classified into homozygous deletion, heterozygous deletion, normal copy, low-level amplification, and high-level amplification. We used homozygous/heterozygous deletions and low/highlevel amplifications unless otherwise stated (e.g., hemizygous deletion). Immunohistochemistry data for estrogen receptor, progesterone receptor, and human epidermal growth receptor-2 were available for 88 samples. The triple-negative subtype was 
determined by each sample's status for estrogen receptor, progesterone receptor, and human epidermal growth receptor-2.

The prediction of the dependencies of the TCGA samples was performed using in silico CRISPR/RNAi based on the Bayesian network. We used the 113 sample for which matched normal transcriptomes were available. We compared the prediction scores from the DNN model, random forest, support vector machine with radial basis function or linear kernels, naïve Bayes classifier, and linear discriminant analysis. Tumor-specific dependencies were identified when the DNN prediction score was greater than 0.5 for the tumor sample and lower than 0.5 for the matched normal sample.

\section{Analysis of dependency-associated somatic alterations}

We collected known oncogenes and tumor suppressor genes (TSGs) from the Cancer Gene Census database [49] and based on the 20/20 rule [50]. In addition to the known cancer genes, we benchmarked the 20/20 rule to discover putative oncogenes and TSGs in breast cancer. For this purpose, we used somatic variant calls from whole-exome sequencing for 988 breast cancer samples. We first catalogued a set of inactivation mutations, which were defined as nonsense mutations or frameshift insertions and deletions. Genes carrying the inactivation mutations in five or more breast cancer samples were regarded as putative TSGs. The known TSGs and putative TSGs were combined and referred to as TSGs in the "Results" section. Putative oncogenes were identified based on missense variants. Specifically, genes carrying the same substitution at the identical position in two or more samples were regarded as potential oncogenes in breast cancer.

For the analysis of dependency-associated mutations, we used somatic variant calls for the 110 tumors that came with tumor and matched normal transcriptomes. For loss-of-function (LOF) mutations, we used nonsense mutations and frameshift insertions or deletions. To detect heterozygous LOF (hetLOF) mutations, we identified heterozygous calls with the variant allele frequency $<40 \%$. Homozygous calls or heterozygous calls with the variant allele frequency $>50 \%$ were regarded as homozygous LOF (homoLOF) mutations. Gain-of-function (GOF) mutations were defined as the recurrent missense variants that we detected to identify the putative oncogenes as described above. The known oncogenes that carried silent mutations only were used as negative controls. For this purpose, we excluded the known oncogenes that have any nonsynonymous mutations in them. The effect of copy number alterations was examined after filtering genes that carry any LOF or GOF mutations in them. The effect of copy number amplifications and silent mutations was examined for the known oncogenes while excluding the putative oncogenes.

\section{Construction of CRISPR/Cas9 vector and Cas9-expressing cells}

Lentiviral guide RNA plasmids were constructed with the pLKO.sgRNA.EFS.tRFP (Addgene \#57823) backbone. Two small guide RNAs (sgRNAs) were designed for each of RAN, XRCC6, and PSMB4 and were inserted into the vector using BsmB I. The two sgRNAs were used simultaneously except for the functional studies of RAN and PSMB4 knockout. The sequences of the sgRNAs used are in Additional file 8: Table S7. 
To produce Cas9-expressing lentiviruses, HEK 293T cells in 100-mm plates with 70\% confluency were co-transfected with packaging plasmids pMD2.G $(10 \mu \mathrm{g})$ and psPAX2 $(10 \mu \mathrm{g})$, lentiCas9-Blast (Addgene \#82372), and pLKO.sgRNA.EFS.tRFP (Addgene \#57823), using the calcium-phosphate transfection method. After $12 \mathrm{~h}$, medium was changed to DMEM medium with 10\% FBS and 1\% PS. Every $12 \mathrm{~h}$ thereafter, the culture supernatant containing the viral particles was harvested and subjected to centrifugation at $1700 \mathrm{rpm}$ at $4{ }^{\circ} \mathrm{C}$ for $10 \mathrm{~min}$ so as to remove any remaining HEK 293T cells.

To generate primary breast cancer cells that stably express Cas 9 , the culture supernatant containing the viruses was supplemented with polybrene $(1 \mu \mathrm{g} / \mathrm{ml})$ and administered to cancer cells for $12 \mathrm{~h}$. This process was repeated three times. After 24-h incubation in fresh RPMI media, the stable Cas9-expressing cells were obtained by selection with $4 \sim 8 \mu \mathrm{g} / \mathrm{ml}$ of Blasticidine S hydrochloride (Sigma).

\section{Knockdown in patient-derived primary cells}

Patient-derived primary cells were cultured in RPMI media containing 5\% FBS, 1\% penicillin/streptomycin, hEGF, hydrocortisone, and transferrin. Viruses with control- or target gene-sgRNA were prepared and applied to infect the primary cancer cells as described above for the construction of Cas9-expressing cells. To confirm knockdown of target genes, cells were harvested $24 \mathrm{~h}$ after the last viral treatment and analyzed by the realtime PCR. Briefly, total RNA extraction was performed using TRizol (Invitrogen). Next, $1 \mu \mathrm{g}$ of total RNA was subjected to cDNA synthesis (PrimeScript RT reagent kit, Cat No. PR037A, Takara). The primer sequence for each target is as follows: Cas9 (forward 5'TTGAAAGGAGTGGGAATTGG-3', reverse 5-'CACGGCGACTACTGCACTTA-3'), XRCC6 (forward 5'-CTTTGAGGAATCCAGCAAGC-3', reverse 5'-ATTGGAGGAG GCTTGAGAGC-3'), RAN (forward 5' -TGTTCCACACCAACAGAGGA-3', reverse 5' TGTTGCCACACAACACAATG-3'), and PSMB4 (forward 5'-GCCAGATGGTGATT GATGAG-3', reverse 5'-CATCGGAGGCTATGCTGATG-3'). Relative expression value was normalized by hRPL13a.

\section{BrdU incorporation and Annexin $\mathrm{V}$ apoptosis assay}

To examine cell cycle progression, the patient-derived cells transfected with the CRISPR/Cas9 lentiviral vector were seeded onto a 6 well-plate. After $24 \mathrm{~h}$, the cells were infected with lentiviruses expressing either control (pLKO5-tRFP; Addgene 57823) or sgRNAs (pLKO5-sgRNAs-tRFP). At each time point (3, 5, or 7 days after lentiviral transfection), $10 \mu \mathrm{M}$ of BrdU was added and incubated for $6-8 \mathrm{~h}$. In vitro BrdU kit (BD Pharmingen, San Diego, CA) was used as described by the manufacturer. Briefly, the cells were fixed with ethanol-containing fixation buffer and washed with the Perm/Wash buffer. For BrdU staining, cells were labeled by the FITC-conjugated antiBrdU antibody for $20 \mathrm{~min}$ at room temperature. After incubation, cells were washed and resuspended with the FACS staining buffer. Finally, FITC (BrdU) and RFP (sgRNA)-positive cells were measured using AccuriFlow Cytometry (BD Biosciences). The percentage of the FITC and RFP-positive cells was calculated using the CFlow software. For apoptosis assay, the cells were seeded onto a 6-well plate as mentioned above. Apoptotic cells were measured by the FITC-Annexin V Apoptosis Detection Kit (BD Pharmingen) at the same time point following the instructions of the manufacturer. 
Briefly, cells were incubated with FITC-Annexin V in the FACS staining buffer for 15 min. The stained cells were immediately subjected to the CFlow software analyses using AccuriFlow Cytometry (BD Biosciences).

\section{HR activity assays for XRCC6-dependent cells}

The PD(+/-)XRCC6 cells transfected with the CRISPR/Cas9 lentiviral vector were infected with lentiviruses expressing either control (pLKO5-tRFP; Addgene 57823) or sgXRCC6 (pLKO5-sgXRCC6-tRFP). After 12-16 h, the cells were transfected with Lipofectamine 2000 as recommended by the manufacturer (Invitrogen) with circular pDR-GFP (Addgene 26,475). After $24 \mathrm{~h}$, the cells were serially transfected with I-SceI producing plasmid pCBASceI (Addgene 26477). After additional $24 \mathrm{~h}$, the GFP-positive cells were quantified on AccuriFlow Cytometry (BD Biosciences). The average HR frequency of three experiments was measured.

The PD(+/-)XRCC6 cells infected with lentiviruses expressing either control or sgXRCC6 (including CRISPR/Cas9) were grown overnight on coverslips and then treated with $\gamma$-irradiation (2Gy). The cells were then fixed with $4 \%$ formaldehyde, permeabilized with $0.1 \%$ Triton $\mathrm{X}-100$, and blocked with $3 \%$ BSA in PBS at room temperature for $1 \mathrm{~h}$. We labeled the cells with primary antibodies for Rad51 (1:500; Abcam) or Ku70 (1200, Santa Cruz), and then stained them with the corresponding Alexa Fluor 488-conjugated secondary antibody (Invitrogen, Carlsbad, CA, USA) for 1 $h$ at room temperature. DAPI was used for nuclear staining. The cells were then mounted with Aqua-Poly-Mount mounting medium and imaged using an inverted fluorescence microscope (Carl Zeiss AG, Oberkochen, Germany). Nuclear Rad51 foci were counted within a region containing at least 50 nuclei by using local maxima in fluorescence intensity.

\section{EGFR nuclear localization in RAN-dependent cells}

The $\mathrm{PD}(+) \mathrm{RAN}$ cells were cultured in RPMI media containing 5\% FBS, 1\% penicillin/ streptomycin, hEGF, hydrocortisone, and transferrin. Viruses for control sgRNA, sgRAN-1, or sgRAN-2 were treated three times at the intervals of $12 \mathrm{~h}$. For EGFR stimulation, the cells were starved overnight with serum-free media. After hEGF treatment $(100 \mathrm{ng} / \mathrm{ml})$, the cells were harvested at $2 \mathrm{~h}$ and $24 \mathrm{~h}$ for western blotting of pEGFR and EGFR. Briefly, the cells were harvested by PBS-EDTA ( $5 \mathrm{mM})$ and centrifuged at $6000 \mathrm{rpm}$ for $5 \mathrm{~min}$. After discarding supernatant, the extraction of nuclear and cytoplasm proteins was carried out following the manufacturer's protocol (Thermo Scientific, \#78833 kit). 10 50 $\mu \mathrm{g}$ of proteins were separated on SDS PAGE, transferred to a nitrocellulose membrane, and probed with antibodies for pEGFR, Cox2, and CyclinD1 (1:1000; Cell Signaling Technology). The membranes were then stripped and reprobed with antibodies for GAPDH (1:1000; Santa Cruz Biotechnology) and Lamin A/C (1:1000; Santa Cruz Biotechnology) to ensure equal loading and fractionation of nucleus and cytoplasm.

\section{Western blot assays}

The PD(+)PSMB4 cells were harvested 48 72 $\mathrm{h}$ after the infection of viruses for control sgRNA, sgPSMB4-1, or sgPSMB4-2. Total protein extract was prepared. Fifteen to 
$30 \mu \mathrm{g}$ of proteins were used per lane. The blot was probed with anti-Cas9 (1:1000; Santa Cruz Biotechnology, sc-517386), anti-PSMB4 (1:500; Santa Cruz Biotechnology, sc-390878), anti-Bad (1:1000; Cell Signaling Technology, ab9239), anti-Bim (1:1000; Cell Signaling Technology, ab2819), and anti-Cytochrome C (1:1000; Abcam, ab13575) antibodies. The $\mathrm{PD}(+) \mathrm{XRCC6}$ and $\mathrm{PD}(-) \mathrm{XRCC} 6$ cells were harvested 1, 3, and 5 days after the infection of viruses for control sgRNA and sgXRCC6. A total of 10-20 $\mu \mathrm{g}$ of proteins was used per lane. The blot was probed with anti-DNA-PKcs (1:200; GeneTex), Ku70 (1:1000; Santa Cruz Biotechnology), and beta-actin (1:1000; Santa Cruz Biotechnology) antibodies. The relative densities of bands were analyzed with the NIIH ImageJ $1.47 \mathrm{v}$ software.

\section{Supplementary information}

Supplementary information accompanies this paper at https://doi.org/10.1186/s13059-020-02077-1.

Additional file 1: Figure S1. Prediction performance by precision and recall. Figure S2. Prediction performance with the ARACNe network. Figure S3. Comparison of simulation results with experimental perturbation data in MCF7. Figure S4. Differential dependency of tumor versus normal samples on genes with copy number changes. Figure S5. Differential dependency of tumor versus normal samples on genes with point mutations. Figure S6. Differential expression of some alternative NHEJ genes in tumor versus normal samples. Figure S7. Additional Annexin $V$ and BrdU data for RAN. Figure S8. Additional Annexin $V$ and BrdU data for XRCC6. Figure S9.

Additional Annexin $V$ and BrdU data for PSMB4. Figure S10. Images of the full uncropped scans for Fig. 7a and Fig. 7b. Figure S11. Increases in the level of proapoptotic proteins by PSMB4 knockout. Figure S12. Changes in DNA-PKCs expression in response to XRCC6 knockdown.

Additional file 2: Table S1. Calibration parameters for deep learning.

Additional file 3: Table S2. Overrepresented function of predicted cancer-specific vulnerabilities in clinical samples.

Additional file 4: Table S3. Common cancer-specific vulnerabilities.

Additional file 5: Table S4. Functional enrichment of overexpressed genes in RAN-dependent samples (CRISPR+RNAi).

Additional file 6: Table S5. Bayesian network in breast cancer.

Additional file 7: Table S6. ARACNe network in breast cancer.

Additional file 8: Table S7. The sequences of the sgRNAs.

Additional file 9. Review history.

Review history

The review history is available as Additional file 9.

Peer review information

Anahita Bishop was the primary editor of this article and managed its editorial process and peer review in collaboration with the rest of the editorial team.

\section{Authors' contributions}

$\mathrm{KJ}$ developed the model and analyzed the data. JSP, HH, and MKS assisted the data analyses. MJP and SC conducted functional experiments. SK, JJ, JWL, and S-YA assisted the functional experiments. $\mathrm{KJ}$ and JKC conceived the study. SC supervised the functional experiments. KJ, MJP, SC, and JKC wrote the manuscript. The authors read and approved the final manuscript.

\section{Funding}

This research was supported by the Bio \& Medical Technology Development Program of the National Research Foundation of Korea funded by the Ministry of Science and ICT (NRF-2017M3A9A7050612 and NRF-

2019M3A9B6064688).

\section{Availability of data and materials}

The codes for in silico CRISPR/RNAi and the deep learning model were made available at GitHub (http://github. com/kaistomics/DeepDependency) [51] and Zenodo (https://zenodo.org/record/3885013, DOl: https://doi.org/10. 5281/zenodo.3885013) [52]. All networks used in this work (the real, shuffled, and inverted Bayesian/ARACNe networks in breast cancer and the Bayesian/ARACNe networks in liver cancer) and the TCGA prediction results are available at http://omics.kaist.ac.kr/resources. We used the ARACNe software available at http://califano.c2b2 columbia.edu/aracne [39]. We downloaded screening results for dependencies from https://depmap.org/portal/ download and https://score.depmap.sanger.ac.uk/downloads. The cancer exome and transcriptome data were obtained from the TCGA database [13]. 
Ethics approval and consent to participate

Not applicable.

Consent for publication

Not applicable.

\section{Competing interests}

The authors declare that they have no competing interests.

\section{Author details}

${ }^{1}$ Department of Bio and Brain Engineering, KAIST, Daejeon 34141, Republic of Korea. ${ }^{2}$ Department of Biomedical Sciences, University of Ulsan College of Medicine, Asan Medical Center, Seoul 05505, Republic of Korea. ${ }^{3}$ Department of Surgery, University of Ulsan College of Medicine, Asan Medical Center, Seoul 05505, Republic of Korea. ${ }^{4}$ Penta Medix Co., Ltd., Seongnam-si, Gyeongi-do 13449, Republic of Korea.

Received: 20 November 2019 Accepted: 18 June 2020

Published online: 27 July 2020

\section{References}

1. Hart T, Chandrashekhar M, Aregger M, Steinhart Z, Brown KR, MacLeod G, et al. High-resolution CRISPR screens reveal fitness genes and genotype-specific cancer liabilities. Cell. 2015;163:1515-26.

2. Wang T, Birsoy K, Hughes NW, Krupczak KM, Post Y, Wei JJ, et al. Identification and characterization of essential genes in the human genome. Science. 2015;350:1096-101.

3. Marcotte R, Sayad A, Brown KR, Sanchez-Garcia F, Reimand J, Haider M, et al. Functional genomic landscape of human breast cancer drivers, vulnerabilities, and resistance. Cell. 2016;164:293-309.

4. Tsherniak A, Vazquez F, Montgomery PG, Weir BA, Kryukov G, Cowley GS, et al. Defining a cancer dependency map. Cell. 2017;170:564-576.e16.

5. McDonald ER, de Weck A, Schlabach MR, Billy E, Mavrakis KU, Hoffman GR, et al. Project DRIVE: a compendium of cancer dependencies and synthetic lethal relationships uncovered by large-scale, deep RNAi screening. Cell. 2017;170:577-592. e10.

6. Wang T, Yu H, Hughes NW, Liu B, Kendirli A, Klein K, et al. Gene essentiality profiling reveals gene networks and synthetic lethal interactions with oncogenic Ras. Cell. 2017;168:890-903.e15.

7. Patel SJ, Sanjana NE, Kishton RJ, Eidizadeh A, Vodnala SK, Cam M, et al. Identification of essential genes for cancer immunotherapy. Nature. 2017;548:537-42.

8. McFarland JM, Ho ZV, Kugener G, Dempster JM, Montgomery PG, Bryan JG, et al. Improved estimation of cancer dependencies from large-scale RNAi screens using model-based normalization and data integration. Nat Commun. 2018;9:4610.

9. Meyers RM, Bryan JG, McFarland JM, Weir BA, Sizemore AE, Xu H, et al. Computational correction of copy number effect improves specificity of CRISPR-Cas9 essentiality screens in cancer cells. Nat Genet. 2017:49:1779-84.

10. Behan FM, lorio F, Picco G, Gonçalves E, Beaver CM, Migliardi G, et al. Prioritization of cancer therapeutic targets using CRISPR-Cas9 screens. Nature. 2019;568(7753):511-6.

11. Kim K, Yang W, Lee KS, Bang H, Jang K, Kim SC, et al. Global transcription network incorporating distal regulator binding reveals selective cooperation of cancer drivers and risk genes. Nucleic Acids Res. 2015;43:5716-29 Available from: http:// nar.oxfordjournals.org/lookup/doi/10.1093/nar/gkv532.

12. Basso K, Margolin AA, Stolovitzky G, Klein U, Dalla-Favera R, Califano A. Reverse engineering of regulatory networks in human B cells. Nat Genet. 2005;37:382-90.

13. The Cancer Genome Atlas Network. Comprehensive molecular portraits of human breast tumours. Nature. 2012;490:6170.

14. Jang K, Kim K, Cho A, Lee I, Choi JK. Network perturbation by recurrent regulatory variants in cancer. PLoS Comput Biol. 2017;13:e1005449 Kann MG, editor. Public Library of Science.

15. Hart T, Moffat J. BAGEL: a computational framework for identifying essential genes from pooled library screens. BMC Bioinformatics. 2016;17:164.

16. Marcotte R, Brown KR, Suarez F, Sayad A, Karamboulas K, Krzyzanowski PM, et al. Essential gene profiles in breast, pancreatic, and ovarian cancer cells. Cancer Discov. 2012;2:172-89.

17. Chen EY, Tan CM, Kou Y, et al. Enrichr: interactive and collaborative HTML5 gene list enrichment analysis tool. BMC Bioinformatics. 2013;14:128. https://doi.org/10.1186/1471-2105-14-128. Published 2013 Apr 15.

18. Eisenberg E, Levanon EY. Human housekeeping genes, revisited [published correction appears in Trends Genet. 2014 Mar;30(3):119-20]. Trends Genet. 2013;29(10):569-74. https://doi.org/10.1016/j.tig.2013.05.010.

19. Hart T, Brown KR, Sircoulomb F, Rottapel R, Moffat J. Measuring error rates in genomic perturbation screens: gold standards for human functional genomics. Mol Syst Biol. 2014;10:733.

20. Xia F, Lee CW, Altieri DC. Tumor cell dependence on Ran-GTP-directed mitosis. Cancer Res. 2008:68:1826-33.

21. Nijhawan D, Zack TI, Ren Y, Strickland MR, Lamothe R, Schumacher SE, et al. Cancer vulnerabilities unveiled by genomic loss. Cell. 2012;150(4):842-54.

22. Weinstein IB, Joe A. Oncogene addiction. Cancer Res. 2008;68(9):3077-80.

23. O'Neil NJ, Bailey ML, Hieter P. Synthetic lethality and cancer. Nat Rev Genet. 2017;18:613-23 Nature Publishing Group.

24. Ly TK, Wang J, Pereira R, Rojas KS, Peng X, Feng Q, et al. Activation of the ran GTPase is subject to growth factor regulation and can give rise to cellular transformation. J Biol Chem. 2010;285(8):5815-26.

25. Spagnolo L, Rivera-Calzada A, Pearl LH, Llorca O. Three-dimensional structure of the human DNA-PKcs/Ku70/Ku80 complex assembled on DNA and its implications for DNA DSB repair. Mol Cell. 2006;22(4):511-9.

26. Lee KY, Im J-S, Shibata E, Dutta A. ASF1a promotes non-homologous end joining repair by facilitating phosphorylation of MDC1 by ATM at double-strand breaks. Mol Cell. 2017;68:61-75.e5 Elsevier. 
27. Dietlein F, Thelen L, Reinhardt HC. Cancer-specific defects in DNA repair pathways as targets for personalized therapeutic approaches. Trends Genet. 2014;30(8):326-39.

28. Dietlein $F$, Thelen $L$, Jokic M, Jachimowicz RD, Ivan L, Knittel G, et al. A functional cancer genomics screen identifies a druggable synthetic lethal interaction between MSH3 and PRKDC. Cancer Discov. 2014;4(5):592-605.

29. Jung J, Jang K, Ju JM, Lee E, Lee JW, Kim HJ, et al. Novel cancer gene variants and gene fusions of triple-negative breast cancers (TNBCs) reveal their molecular diversity conserved in the patient-derived xenograft (PDX) model. Cancer Lett. 2018:428:127-138.

30. Ran FA, Hsu PD, Wright J, Agarwala V, Scott DA, Zhang F. Genome engineering using the CRISPR-Cas9 system. Nat Protoc. 2013;8:2281-308.

31. Han W, Lo H-W. Landscape of EGFR signaling network in human cancers: biology and therapeutic response in relation to receptor subcellular locations. Cancer Lett. 2012;318:124-34.

32. Dittmann K, Mayer C, Fehrenbacher B, Schaller M, Kehlbach R, Rodemann HP. Nuclear EGFR shuttling induced by ionizing radiation is regulated by phosphorylation at residue Thr654. FEBS Lett. 2010;584:3878-84.

33. Pierce AJ, Johnson RD, Thompson LH, Jasin M. XRCC3 promotes homology-directed repair of DNA damage in mammalian cells. Genes Dev. 1999;13:2633-8.

34. Bryant HE, Schultz N, Thomas HD, Parker KM, Flower D, Lopez E, et al. Specific killing of BRCA2-deficient tumours with inhibitors of poly (ADP-ribose) polymerase. Nature. 2005;434(7035):913-7.

35. Farmer H, McCabe N, Lord CJ, et al. Targeting the DNA repair defect in BRCA mutant cells as a therapeutic strategy. Nature. 2005;434(7035):917-921. https://doi.org/10.1038/nature03445.

36. Fong PC, Boss DS, Yap TA, Tutt A, Wu P, Mergui-Roelvink M, et al. Inhibition of poly (ADP-ribose) polymerase in tumors from BRCA mutation carriers. N Engl J Med. 2009;361:123-34.

37. Gurley KE, Kemp CJ. Synthetic lethality between mutation in Atm and DNA-PK (cs) during murine embryogenesis. Curr Biol. 2001;11:191-4 Elsevier.

38. Riabinska A, Daheim M, Herter-Sprie GS, Winkler J, Fritz C, Hallek M, et al. Therapeutic targeting of a robust nononcogene addiction to PRKDC in ATM-defective tumors. Sci Transl Med. 2013;5(189):189ra78.

39. Margolin AA, Nemenman I, Basso K, Wiggins C, Stolovitzky G, Favera R, et al. ARACNE: an algorithm for the reconstruction of gene regulatory networks in a mammalian cellular context. BMC Bioinformatics. BioMed Cent; 2006;7: S7.

40. Reynolds A, Leake D, Boese Q, Scaringe S, Marshall WS, Khvorova A. Rational siRNA design for RNA interference. Nat Biotechnol. 2004;22:326-30.

41. Bergstra J, Breuleux O, Bastien FF, Lamblin P, Pascanu R, Desjardins G, et al. Theano: a CPU and GPU math compiler in Python. Proc 9th Python Sci Conf (SCIPY 2010). 2010;

42. Erhan D, Courville A, Vincent P. Why does unsupervised pre-training help deep learning? J Mach Learn Res. 2010;11: 625-60.

43. Zou H, Hastie T. Regularization and variable selection via the elastic net. J R Stat Soc Ser B Stat Methodol. 2005;67:30120.

44. Sutskever I, Martens J, Dahl GE, Hinton GE. On the importance of initialization and momentum in deep learning. Jmlr W\&Cp. 2013;28:1139-47.

45. Liaw A, Wiener M. Classification and regression by randomForest. R News. 2002;2:18-22.

46. Meyer D, Dimitriadou E, Hornik K, Weingessel A, Leisch F, Chang C-C, et al. Misc functions of the Department of Statistics, Probability Theory Group, TU Wien. R Packag. version 1.6-2. 2015. https://cran.r-project.org/web/packages/e1 071/index.html.

47. Venables WN, Ripley BD. Modern applied statistics with S Fourth edition by. World; 2002

48. Mermel CH, Schumacher SE, Hill B, Meyerson ML, Beroukhim R, Getz G. GISTIC2.0 facilitates sensitive and confident localization of the targets of focal somatic copy-number alteration in human cancers. Genome Biol. 2011;12(4):R41.

49. Futreal PA, Coin L, Marshall M, Down T, Hubbard T, Wooster R, et al. A census of human cancer genes. Nat Rev Cancer. 2004;:177-83.

50. Vogelstein B, Papadopoulos N, Velculescu VE, Zhou S, Diaz LA Jr, Kinzler KW. Cancer genome landscapes. Science. 2013; 339(6127):1546-58. https://doi.org/10.1126/science.1235122.

51. Jang K, Park MJ, Park JS, Hwangbo H, Sung MK, Kim S, Jung J, Lee JW, Ahn S-Y, Chang S, Choi JK. Computational inference of cancer-specific vulnerabilities in clinical samples. GitHub. http://github.com/kaistomics/DeepDependency (2020).

52. Jang K, Park MJ, Park JS, Hwangbo H, Sung MK, Kim S, Jung J, Lee JW, Ahn S-Y, Chang S, Choi JK. Computational inference of cancer-specific vulnerabilities in clinical samples. Zenodo. https://doi.org/10.5281/zenodo.3885013 (2020).

\section{Publisher's Note}

Springer Nature remains neutral with regard to jurisdictional claims in published maps and institutional affiliations.

Ready to submit your research? Choose BMC and benefit from:
- fast, convenient online submission
- thorough peer review by experienced researchers in your field
- rapid publication on acceptance
- support for research data, including large and complex data types
- gold Open Access which fosters wider collaboration and increased citations
- maximum visibility for your research: over 100M website views per year
At BMC, research is always in progress.
Learn more biomedcentral.com/submissions

